reduction in ambulatory adults seeking care for bronchitis in our health system and to a dramatic reduction in antibiotic prescribing for that condition. Reducing unnecessary, outpatient antibiotic prescribing has long been a difficult challenge for many antimicrobial stewardship programs. Our challenge will be to educate patients and clinicians to maintain these improvements in outpatient, acute bronchitis management as the pandemic is brought under control and outpatient visits return to prepandemic levels.

\section{References}

1. Abelenda-Alonso G, Padullés A, Rombauts A, et al. Antibiotic prescription during the COVID-19 pandemic: a biphasic pattern. Infect Control Hosp Epidemiol 2020. doi: 10.1017/ice.2020.381.

2. Nestler M, Godbout E, Lee K, et al. Impact of COVID-19 on pneumoniafocused antibiotic use at an academic medical center. Infect Control Hosp Epidemiol 2020. doi: 10.1017/ice.2020.362.

3. Jeffery MM, D'Onofrio G, Paek $\mathrm{H}$, et al. Trends in emergency department visits and hospital admissions in health care systems in 5 states in the first months of the COVID-19 pandemic in the US. JAMA Intern Med 2020 Aug 3 [Epub ahead of print]. doi: 10.1001/jamainternmed.2020. 3288.

4. Pagels CM, Dilworth TJ, Fehrenbacher L, Singh M, Brummitt CF. Impact of an electronic best-practice advisory in combination with prescriber education on antibiotic prescribing for ambulatory adults with acute, uncomplicated bronchitis within a large integrated health system. Infect Control Hosp Epidemiol 2019;40:1348-1355.

5. Nolen LD, Seeman S, Bruden D, et al. Impact of social distancing and travel restrictions on non-COVID-19 respiratory hospital admissions in young children in rural Alaska. Clin Infect Dis 2020 [Epub ahead of print]. doi: 10.1093/cid/ciaal328peds.

6. Hatoun J, Correa ET, Donahue SMA, Vernacchio L. Social Distancing for COVID-19 and Diagnoses of Other Infectious Diseases in Children Pediatrics. 2020 Sep 2 [Epub ahead of print]. doi: 10.1542/peds. 2020-006460.

7. Harris AM, Hicks LA, Qaseem A. Appropriate antibiotic use for acute respiratory tract infection in adults. Ann Intern Med 2016;165:674.

\title{
Implementation of COVID-19 Provider Resource Task Force: A provider support initiative during emergency preparedness in a quaternary-care center in western Michigan
}

\author{
Vetriselvi Moorthy $\mathrm{MD}^{1}$ (1), Mudita Bhugra $\mathrm{MD}^{1}$, Curtis J. Behenna $\mathrm{DO}^{1}$, Manivannan Veerasamy $\mathrm{MD}^{2}$, \\ Michael J. Harrison $\mathrm{MD}^{3}$, Liam R. Sullivan $\mathrm{DO}^{1}$, Russell J. Lampen $\mathrm{DO}^{1}$, Habiba Hassouna $\mathrm{MD}^{1}$ \\ and Jorgelina T. de Sanctis MD ${ }^{1}$ \\ ${ }^{1}$ Spectrum Health Infectious Diseases/Michigan State University, Grand Rapids, Michigan, ${ }^{2}$ Spectrum Health Richard McNamara Cardiovascular Diseases \\ Fellowship/Michigan State University, Grand Rapids, Michigan and ${ }^{3}$ Specialties/Digestive Health, Spectrum Health Medical Group, Grand Rapids, Michigan
}

To the Editor-On March 11, 2020, World Health Organization (WHO) announced coronavirus disease 2019 (COVID-19) a pandemic. ${ }^{1}$ Spectrum Health System, like many other healthcare systems, activated an emergency preparedness command center and implemented strategies to mitigate the impact of COVID-19. Spectrum Health System is a nonprofit quaternary-care health system based in Grand Rapids, Michigan. More than 4,600 physicians and advanced-practice providers offer care to patients through 14 hospitals, including Helen DeVos Children's Hospital and 150 ambulatory clinics. Grand Rapids medical community also includes 2 other health systems, many ambulatory specialty and subspecialty practices, and rehabilitation units. During the COVID-19 pandemic, Spectrum Health initiated a work group as a support initiative for the patient-facing clinicians in the entire community.

\section{Methods}

The COVID-19 Provider Resource Work Group and hotline were commissioned on March 19, 2020. The aims of the work group

Author for correspondence: Vetriselvi Moorthy, 230 Michigan St NE, Grand Rapids, Michigan 49503. E-mail: Vetriselvi.moorthy@spectrumhealth.org

Cite this article: Moorthy V, et al. (2021). Implementation of COVID-19 Provider Resource Task Force: A provider support initiative during emergency preparedness in a quaternary-care center in western Michigan. Infection Control \& Hospital Epidemiology, 42: 1292-1294, https://doi.org/10.1017/ice.2020.1247 were (1) to provide real-time provider-to-provider education regarding questions that pertain to COVID-19; (2) to respond to a COVID-19 e-mail inbox that received questions from 31,000 employees within the Spectrum Health System; (3) to enable triage providers to direct their patients to appropriate channels; and (4) to improve provider access to updates on testing, treatment guidelines, and web-based resources. In total, 18 Spectrum Health physicians from infectious disease, allergy and immunology, internal medicine, and pulmonary critical care, as well as infectious disease fellow physicians volunteered to be part of the work group.

Initially, clinical questions from all the providers across the medical community would go through the provider hotline. This hotline was attended by a nurse who directed the appropriate calls to the on-call physician from the work group. Physicians self-assigned themselves to 4-hour shifts 7 days a week from 8 A.M. to 9 P.M. on a secure HIPAA-compliant communication platform called Perfect Serve. A shared inbox "covid19dr" was attached to the physicians' personal organization e-mail to receive questions. After an initial meeting that was organized to review the goals and objectives, the work group communicated daily to discuss the challenges, work flow, and current literature. Physicians self-educated on the current literature by reviewing Spectrum Health LitCovid, an internally created database maintained by

(c) The Author(s) 2020. This is an Open Access article, distributed under the terms of the Creative Commons Attribution licence (http://creativecommons.org/licenses/by/4.0/), which permits unrestricted re-use, distribution, and reproduction in any medium, provided the original work is properly cited. 
Table 1. Spectrum of Calls and E-mails Received by the Provider Resource Work Group

\begin{tabular}{|c|c|}
\hline Period & Types of Calls and E-mails \\
\hline Week 1 & $\begin{array}{l}\text { - Use of PPE with negative COVID-19 testing and removing isolation precautions } \\
\text { - Asthma exacerbation and use of nebulizers with positive COVID-19 test } \\
\text { - Appropriate N-95 mask use for all providers } \\
\text { - Appropriate timing to initiate hydroxychloroquine } \\
\text { - Encephalopathy and fever, is that COVID-19 presenting symptom? } \\
\text { - Sensitivity of COVID-19 testing } \\
\text { - Many outpatient offices with question about testing versus self-quarantine at home due to limited testing } \\
\text { - Oncology offices with questions about COVID-19 testing prior to chemotherapy; recent travel, cough and need for testing; } \\
\text { diarrhea in bone marrow transplant patient and COVID-19 testing }\end{array}$ \\
\hline Week 2 & $\begin{array}{l}\text { - Help with completion of COVID-19 testing paperwork for the health department } \\
\text { - Fit testing for N-95 masks } \\
\text { - Provider unclear on duration of quarantine whether } 7 \text { or } 14 \text { days } \\
\text { - Return to work after COVID-19 } \\
\text { - Patient coughed on providers before getting COVID-19 test, significant exposure or not and enquired the next step } \\
\text { - No viral swab available for other viral testing, what is the next step? }\end{array}$ \\
\hline Week 3 & $\begin{array}{l}\text { - Visitor exception for end of life patient with negative COVID-19 test } \\
\text { - Routine preoperative testing for COVID-19 } \\
\text { - Deployment for a retired physician who wishes to volunteer for COVID-19 } \\
\text { - COVID-19 testing prior to discharge to rehabilitation centers } \\
\text { - Questions about convalescent plasma infusion and accessibility } \\
\text { - Queries received about immunomodulator use in elderly patients } \\
\text { - Private outpatient offices sent queries about referring COVID-19 testing to the Spectrum Health laboratory } \\
\text { - Can oropharyngeal swab be used for COVID-19 testing? } \\
\text { - CPAP use in COVID-19 patients }\end{array}$ \\
\hline Week 4 & $\begin{array}{l}\text { - Whether operation room is open for elective cases } \\
\text { - What is the next step if patients or family refuse to wear mask? } \\
\text { - Guidance on remdesivir and tocilizumab therapy } \\
\text { - Provider failed N-95 mask fit test } \\
\text { - Cost and availability of COVID-19 antibody testing for providers } \\
\text { - Repeat COVID-19 testing positive after } 1 \text { month and what is the appropriate time for plasma donation? } \\
\text { - Families of patients hospitalized for other illnesses requested COVID-19 testing prior to discharge }\end{array}$ \\
\hline
\end{tabular}

Note. PPE, personal protective equipment; CPAP, continuous positive airway pressure.

the research department. No additional financial incentive was provided to the physicians; participation was voluntary.

The calls and e-mails were tracked. The daily number of secure messages ranged from 10 to 20 during the first 4 weeks and progressively diminished over the following weeks when providers were more familiar with the process. The daily e-mails numbered 5-10 initially, followed by a steady decrease. Resources and guidelines were updated in real time within the organization website. Physicians documented the questions and the actions taken so they could be discussed at the daily meeting. Calls were escalated to the infectious disease on-call provider if necessary.

\section{Analysis}

The medical community of Grand Rapids, Michigan, like others across the world, was facing uncertainties regarding the novel nature of SARS CoV-2 virus, limited treatment options, shortage of personal protective equipment (PPE), and inadequate testing supplies. ${ }^{2}$ Our provider work group played a tremendous role in the psychosocial well-being of the clinicians by aiding with both personal and professional responsibilities and addressing concerns. ${ }^{2}$

Providers from intensive care unit, inpatient medical units, ambulatory centers, rehabilitation units, hospice unit, outside hospital, and private pediatric and obstetrics offices reached out to the work group. The spectrum of concerns raised included guidance on testing, PPE, isolation precautions, immunocompromised patients, supply chain, and treatment options (Table 1).

As we gained more experience with COVID-19 patient care and robust testing and we increased availability of PPE and developed protocols, the number of calls and e-mails steadily decreased, indicating that the clinicians rapidly adapted themselves, mastered new skills, and revised practices. With that decrease, meetings were changed to every other day and then were conducted weekly. The work group was concluded 8 weeks later when the providers were more confident with everyday patient care, appropriate testing criteria, and PPE use for COVID-19 patients.

\section{Discussion}

The medical community has come together and responded in a heroic fashion to the challenges posed by the COVID-19 pandemic. ${ }^{3}$ Spectrum Health's COVID-19 Provider Resource Work Group hotline is one of the many efforts instituted to improve COVID-19 patient care and control the spread in both hospital and ambulatory settings. Concerns from the work group were appropriately streamlined to the respective COVID-19 performance committee. Other quality improvement initiatives were conducted based on these concerns to restructure the work flow and to improve patient care. By making the COVID-19 provider 
hotline accessible to all providers across the community, Spectrum Health prioritized the professional development of healthcare workers as well as their psychosocial well-being during the pandemic.

Frontline healthcare staff are not only experiencing a rapid increase in the volume and intensity of their work but are also facing additional challenges such as unfamiliar working environments, changing protocols, and unprecedented exposure to COVID-19 with little opportunity for orientation and training. ${ }^{4}$ It is widely recognized that healthcare professionals need evidence-based support initiatives to mitigate the effects of COVID-19 on their current and future well-being. ${ }^{4}$ Spectrum Health's COVID-19 Provider Resource Work Group is an example of a successful and crucial support initiative in closing the knowledge gap and having a positive impact on the providers, emphasizing the need for support resources during these tumultuous times. Similar support initiatives and resources should be made available for clinicians during any other healthcare emergency or outbreak that has a public health impact.

\section{Acknowledgments.}

Financial support. No financial support was provided by Spectrum Health or any other entity for the preparation of this article.

Conflicts of interest. Authors of this article have no relevant conflicts of interest to disclose.

\section{References}

1. Cucinoto D, Vanelli M. WHO declares COVID-19 a pandemic. Acta Biomed 2020;91:157-160.

2. Pfefferbaum B, North CS. Mental health and the COVID-19 pandemic. N Engl J Med 2020;383:510-512.

3. Emmanuel A. COVID-19: the physician's response in the first phase. Clin Med (Lond) 2020;20:237.

4. Kinman G, Teoh K, Harriss A. Supporting the well-being of healthcare workers during and after COVID-19. Occupat Med 2020;70:294-296.

\title{
Regional outbreak of methicillin-resistant Staphylococcus aureus ST2725-t1784 in rural Japan
}

\author{
Satoru Mitsuboshi $\mathrm{PhD}^{1}$ (1), Toshio Yamaguchi $\mathrm{PhD}^{2}$, Hyuji Seino², Masahiro Fukuhara $\mathrm{PhD}^{2}$, Yasuka Hosokawa ${ }^{3}$ and \\ Masami Tsugita PhD 4 \\ ${ }^{1}$ Department of Pharmacy, Kaetsu Hospital, Niigata, Japan, ${ }^{2}$ Department of Microbiology, Niigata University of Pharmacy and Applied Life Sciences, Niigata, \\ Japan, ${ }^{3}$ Department of Pharmacy, Niitsu Medical Center Hospital, Niigata, Japan and ${ }^{4}$ Department of Clinical Pharmacology, Faculty of Pharmaceutical Sciences, \\ Niigata University of Pharmacy and Applied Life Sciences, Niigata, Japan
}

To the Editor-Novel strains of methicillin-resistant Staphylococcus aureus (MRSA) continue to be discovered, ${ }^{1}$ and understanding the trends of these strains in each region is important because MRSA genotype affects clinical outcomes. ${ }^{2}$ Despite the introduction of various interventions, the prevalence of MRSA infection in Japanese hospitals remains relatively high. ${ }^{3}$ Although MRSA USA300, which causes severe infectious disease with high mortality, is not common in Japan, ${ }^{4}$ other SCCmec type IV MRSA strains have become widespread. ${ }^{5}$

Akiha Ward has 3 hospitals serving a population of $\sim 80,000$. The local incidence of MRSA bacteremia was $\sim 10$ per 100,000 person years in 2017-2018, which is considered high. ${ }^{6}$ Therefore, to clarify the main route of MRSA transmission, we surveyed clinical isolates of MRSA from Akiha Ward using whole-genomic sequencing (WGS).

We collected clinical isolates of MRSA from 2 hospitals, namely, Kaetsu Hospital and Niitsu Medical Center Hospital, from April to September 2018. All clinics and a psychiatric hospital were excluded because they performed a relatively low number of

Author for correspondence: Satoru Mitsuboshi, E-mail: ccrtyo34057@gmail.com Cite this article: Mitsuboshi S, et al. (2021). Regional outbreak of methicillin-resistant Staphylococcus aureus ST2725-t1784 in rural Japan. Infection Control \& Hospital Epidemiology, 42: 1294-1296, https://doi.org/10.1017/ice.2020.1265 bacterial tests. Despite these exclusions, our data covered most bacterial tests performed in Akiha Ward and thus can be considered to reflect the local epidemiology of MRSA infection. WGS analyses were performed at the Department of Microbiology, Niigata University of Pharmacy and Applied Life Sciences (NUPALS). The protocol was approved by the institutional review boards of NUPALS (no. H30-05), Kaetsu Hospital (approval \# 2018-2), and Niitsu Medical Center Hospital (no. 2018-2). All clinically characterized MRSA isolates, including redundant ones, were further confirmed using MRSAII selective agar (Eiken-Chemical, Japan) and WGS. Additional isolates from the same patient with the same multilocus sequence typing profile were considered to be redundant samples and were therefore excluded, leaving 120 individual strains from 102 patients for further analysis.

Total DNA samples were prepared from each isolate by DNeasy Ultraclean Microbial kit (Qiagen, Germany), and WGS was performed using NexteraXT and Miseq Reagent kit v2 (500 cycles) or v3 (600 cycles) (Illumina, USA). All read sequences were deposited in the DDBJ/EMBL/GenBank database (accession nos. DRA010434-DRA010440). Multilocus sequence typing (MLST) analysis was performed using nullarbor v.2.0.20180819 (https://github.com/tseemann/nullarbor) with SPAdes v.3.12.0 as the assembler. ${ }^{7}$ A k-mer-based tool, stringMLST, was also used for MLST analysis when the above software failed to define the sequence type. ${ }^{8}$ SCCmec and spa typing were performed by 